\title{
Die kirchliche Publizistik im jüngeren Strukturwandel der Öffentlichkeit
}

Der Bayerische Presseclub feierte am 10. November 2004 in der Katholischen Akademie in München sein fünfzigjähriges Bestehen. Er hat mich gebeten, einen Festvortrag zu halten. Im Unterschied zum sonst üblichen Brauch, Vorträge, wenn sie im Druck publiziert werden sollen, so zu redigieren, dass sie vom wissenschaftlichen Aufsatz nicht zu unterscheiden sind, habe ich in diesem Falle an der Vortragsform weitgehend festgehalten, und zwar aus folgendem Grund: Unter den zuhörenden Clubmitgliedern waren nicht wenige, mit denen ich in jenem halben Jahrhundert das eine oder andere Stück Weges gemeinsam gegangen bin, sei es als Joumalist und Kollege, sei es später als analysierender Wissenschaftler und Berater. Daraus ergab sich ein sehr persönliches Gesprächsklima, und so soll es im folgenden Text auch bleiben.

Einem Emeritus ist es - gelegentlich - erlaubt, mit einer autobiographischen Anekdote zu beginnen. Wer unter den Zuhörern 50 Jahre zurückblicken kann, wird sich auch an den Chefredakteur Dr. Franz Kroos aus Münster in Westfalen erinnern. Dort regierte und redigierte er in autokratischer Manier das Bistumsblatt „Kirche und Leben“. Ab 1957 brachte er auch mir das $A B C$ des Journalismus bei, fromm und streng und eines jeglichen Modernismus gänzlich unverdächtig. Eben dieser erzkonservative Franz Kroos erteilte im Jahre 1968 seinem Volontär Manfred Becker den für damalige Verhältnisse revolutionären Auftrag, die Bistumspresse in der Bundesrepublik Deutschland auf Herz und Nieren zu untersuchen. Becker nahm die Sache ernst, entwickelte aus der empirischen Untersuchung eine Seminararbeit am Institut für Publizistik der Universität Münster und machte offenbar, wovon auf den Tagungen der Arbeitsgemeinschaft Kirchliche Presse schon seit einiger Zeit gemunkelt wurde: Es geht bergab.

\section{Eine vorhersehbare Entwicklung}

Was sich damals allein unter dem Aspekt der Auflagenentwicklung offenbarte, lässt sich am besten optisch darstellen. Die Auflagenkurve der „Münchner Katholischen Kirchenzeitung“" von 1963 bis 1969 sah so aus: 


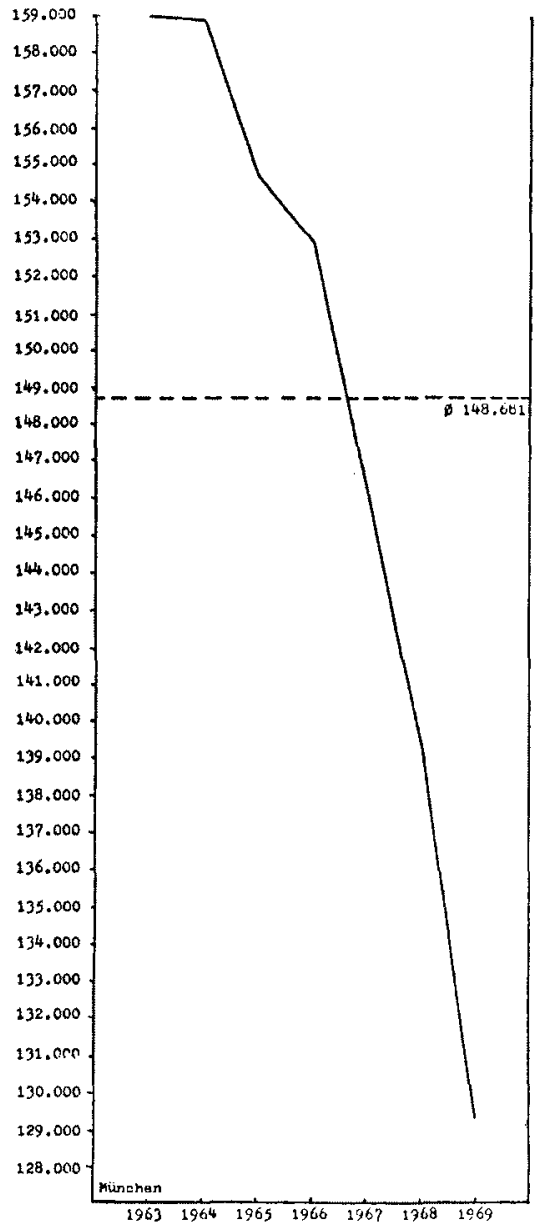

München

"Münchener katholische Kirchenzeitung“ Auflage: 129398

Seitenzahl: 24

Bezugspreis: 1,50

Satzspiegel einer Druckseite: $33,3 / 23$

Druckfläche einer Seite: 765,90

durchschnittliche gesamte Druckfläche: Anzeigenfläche: 18389,60 1182,50 redaktionelle Drudkfläche: 17199,10 Anteil der durchschnittlichen Anzeigenfläche an der durchschnitrlichen Drudkfläche: $6,4 \%$

Es gab auch einige nicht ganz so dramatische Talfahrten im Fähnlein der 22 Aufrechten. Aber es gab nur eine echte Ausnahme: Münster (siehe Abbildung auf der folgenden Seite).

Als die Grafiken (1970) in unserer „Communicatio Socialis“ publiziert wurden ${ }^{1}$, mag sich Franz Kroos gefreut haben - aber man täte ihm unrecht, wollte man ihm unterstellen, er habe die Untersuchung nur angeregt, um den Ruhm des ohnehin Klassenbesten „Kirche und Leben“ zu mehren.

Kroos hatte nicht nur für lokaljournalistische, sondern auch für die

1 Manfred P. Becker: Die Bistumspresse in der Bundesrepublik Deutschland. 1. Folge. In: Communicatio Socialis (ComSoc), 3. Jg. 1970, S. 299-310. 


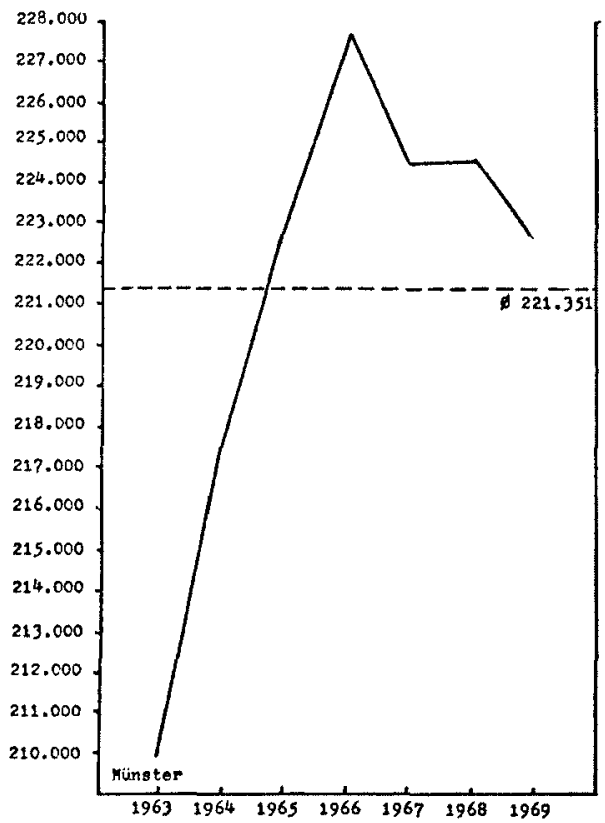

Münster

"Kirche und Leben"

Auflage: 222563

Seitenzahl: 16

Bezugspreis: 1,80

Satzspiegel einer Druckseite: $40 / 27,5$

Druckfläche einer Seite 1100

durchschnitrliche

gesamte Druckfläche:

Anzeigenfläche:

17600,00

2645,75

redaktionelle Druckfäche: 14954,25

Anteil der durchschnitrlichen Anzeigenfläche an der durchschnittlichen Druckfläche: $15,0 \%$

großen Wendegeschichten in Kirche und Gesellschaft ein seismographisches Gespür. In diesem Punkt wurde er belohnt. Der Gesamtüberblick über die Jahre 1950 bis 1969 zeigte: Der große Knick fand 1963 statt, ausgerechnet als das Zweite Vaticanum gerade begonnen hatte. Seither sank nicht nur die Auflage, sondern auch die Bezieherdichte:

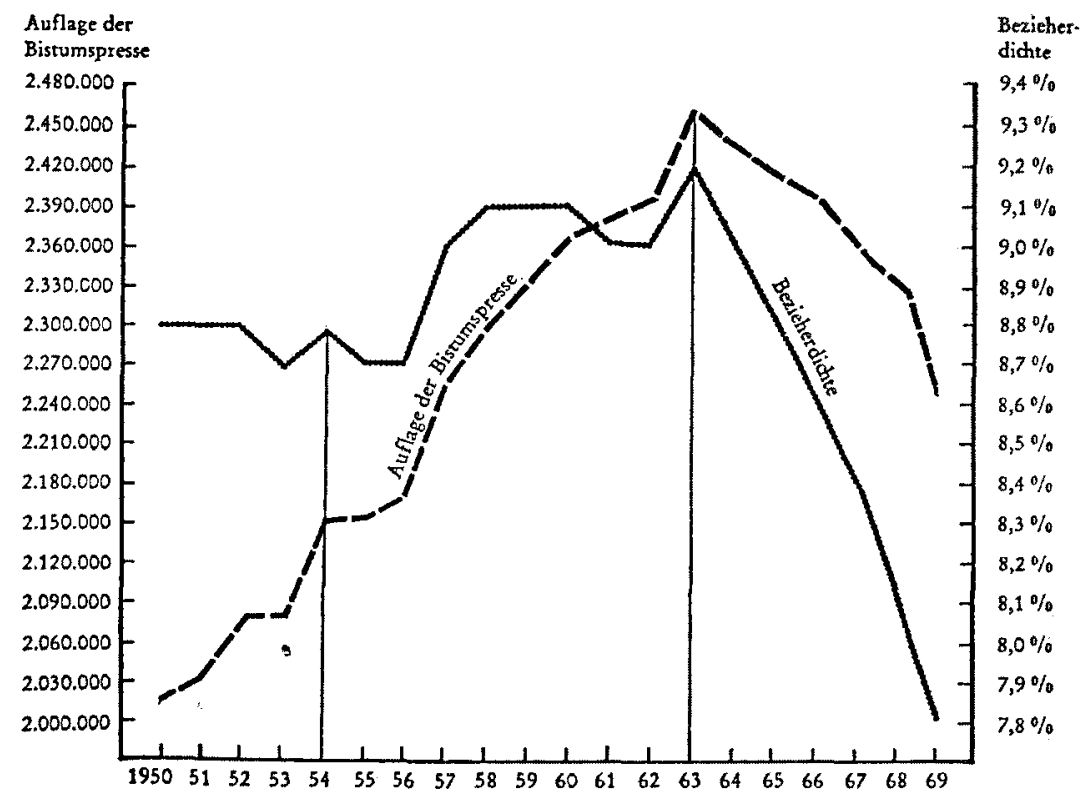


Das heißt im Klartext: Immer weniger Menschen waren bereit, für ihre Kirchenzeitung Geld auszugeben. Nun hätte man das ganze als eine innerkatholische Entwicklung abtun können, hätte sich nicht ein anderes Datum hinzugesellt, das eine gesamtgesellschaftliche Relevanz bekam. Wie schon einige Zeit früher in den evangelischen Kirchen, begann auch in der katholischen Kirche die Zahl der sonntäglichen Kirchenbesucher zu sinken. In einem - bis heute unveröffentlichten - Gutachten für die Deutsche Bischofskonferenz ${ }^{2}$, erstellt in den Jahren 1972/73, setzten wir die Kirchenbesucherkurve in ein zunächst nur optisches Verhältnis zur Auflagen- und zur Bezieherdichten-Kurve der Bistumspresse. Wir haben damals darauf verzichtet, den statistischen Zusammenhang exakt zu überprüfen, und stattdessen gesagt: „Die aufgezeigten Vergleichswerte deuten nicht zwingend auf einen Kausalzusammenhang zwischen dem Rückgang der ,Kirchlichkeit' und dem Sinken der Auflagen, sie legen aber eine entsprechende Hypothese nahe."

Diese Hypothese hat sich nun über die Jahre hin gewissermaßen selbst überprüft, und zu Beginn der 1990er Jahre bestätigte sich die Parallel-Talfahrt der beiden Kurven ${ }^{3}$ :

2 Gutachten Katholische Publizistik 72/73. Auftragnehmer war der Autor dieses Beitrags.

3 Abbildung auf der nächsten Seite, übernommen aus Michael Schmolke: Anfang der Talsohle. In: ComSoc, 26. Jg. 1993, S. 389-393, findet sich - bestätigend fortgesetzt in der Abbildung 1 des in diesem Heft von ComSoc publizierten Beitrags von Helmut Haimerl: Zukunftsfähige Bistumszeitungen. 
Gesamtauflage der Bistumspresse und sonntäglicher Gottesdienstbesuch in den alten Bundesländern 1950 - 1992

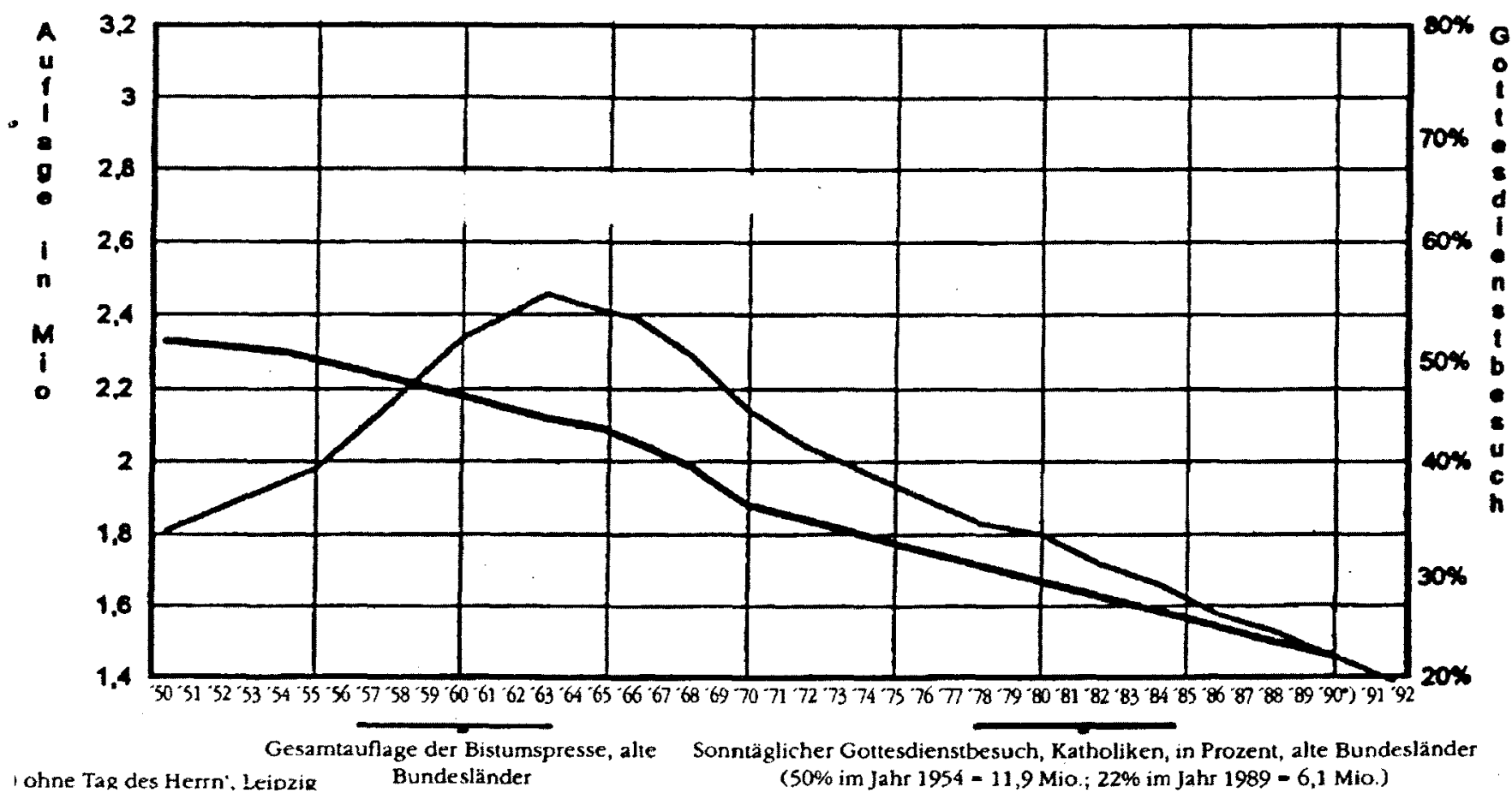


Das heißt aber nichts anderes, als dass man schon 20 Jahre früher, also zur Zeit der Würzburger Synode, ziemlich genau wusste oder hätte wissen können, wie der Hase läuft. Ausgehend von einer „essentiellen Krise" der Bistumspresse gab ich im erwähnten Gutachten zu bedenken, dass es sich um mehr handeln könnte als um einen an verschiedenen Indikatoren ablesbaren „Rückgang der Kirchlichkeit“, gemessen in Gottesdienstbesucherzahlen und Kirchenblattauflagen. „Eine Einbeziehung anderer Indikatoren könnte möglicherweise das Ergebnis bringen, dass es sich hier nicht um einen linearen, eindimensionalen Rückgang von Kirchlichkeit handelt, sondern um einen wesentlichen Wandel im kirchlichen Selbstverständnis des einzelnen ..."4

Die weitere Entwicklung nach 1974, dem Abschlussjahr des Gutachtens, und auch die Maßnahmen, mit denen die offizielle Kirche gegenzusteuern versuchte, haben wir alle miterlebt. In ihrem Verlauf wurde die Kirchenpresse, die mindestens bis zur Würzburger Synode noch ein ganz wichtiger Indikator gewesen war, ein Randthema. Man widmete ihr zwar viel Aufmerksamkeit in Gestalt mehrfacher Feldbefragungen, aber nach deren letzter, der Allensbach-Studie von 1994, durfte man ungestraft sagen: „Seit 30 Jahren Niedergang ohne Untergang“. 5 Selbst die AKP in ihrer klassischen Form verschwand von der Bildfläche.

\section{Die Dialog-Idee in Konzils- und Synodenzeiten}

Schon vor der Würzburger Synode hatte die katholische Intelligentsia erkannt, dass es um etwas anderes ging als um das effektive Funktionieren der publizistischen acies formata, die da bestand aus Kirchenpresse, KNA und den kirchlichen Bastionen in den öffentlich-rechtlichen Rundfunkanstalten.

Es ging um die generelle Teilnahme der Kirche oder doch des deklariert katholischen Bevölkerungsanteils am Dialog der Gesellschaft. Der von Johannes XXIII. ausgehende Anstoß zum Dialog war willkommen und wurde durchaus aufgenommen. Als äußere Zeichen dafür entstanden (1968) sogenannte Dialogzeitschriften: die „Internationale Dialog Zeitschrift" bei Herder und - in Wien - Günther Nennings runderneuertes „Neues Forum“ mit dem Untertitel „Internationale Zeitschrift für den Dialog". Was fehlte, war jedoch das Blatt, das nicht nur den Dialog im Titel führte, sondern den Dialog mit starker Stimme führen konnte. 
Ein Wunder von der Größenordnung, wie sie der jüngst selig gesprochene Kaiser Karl gebraucht hätte, geschah: Ebenfalls 1968 sprang die Deutsche Bischofskonferenz über alle ihre Schatten und gründete „Publik". Aber ehe die katholische Wochenzeitung zur wirklich starken Stimme heranwachsen konnte, wurde sie selbst Dialogthema. Das war gut und richtig so, sage ich, obwohl das Blatt am Ende zu Tode dialogisiert wurde. Schon 1969 bekamen die Bischöfe Angst vor der eigenen Courage und mit dem 19. November 1971 wurde „Publik“ eingestellt. Aber allein die Unzahl der aus allen Lagern kommenden Pressestimmen zur Entstehung und zum Ende der Zeitung dokumentierte, dass hier ein Kernthema des deutschen Nachkriegskatholizismus angerührt worden war. Nach meiner Erinnerung war kein Arbeitstag der Würzburger Synode aufregender und anregender als der „Publiktag“ am 11. Mai 1972 - es kam, wie ich 1976 resümierte, „zu zwar engagierten, aber am Ende für alle frustrierenden Diskussionen: Publizistik als Thema war seither der Synode verleidet." 6

Dessen ungeachtet war die Synode selbst wie zuvor schon das Zweite Vaticanum Gegenstand der allgemeinen öffentlichen Diskussion, und zwar nicht zuletzt wegen des dialogischen Stils beider Ereignisse: Alles hatten die Massenmedien von der katholischen Kirche erwartet, nur nicht einen Papst, der das aggiomamento ankündigte, und Kirchenversammlungen, in denen offen und öffentlich beraten und von denen Beratungsergebnisse demokratischen Abstimmungen unterworfen werden konnten.

Das Unerwartete war in diesem Falle die Mutter der Aktualität. Aber die Aktualität hat viele Mütter.

\section{Behutsamer Rückzug - aber die Kirche bleibt interessant}

Die nachkonziliare Kirche in Deutschland, schreibt Eckhard Bieger, sei u.a. „Opfer des Öffentlichkeitserfolges des Konzils“. ${ }^{7}$ Und ebenso der Synode, füge ich hinzu.

In den Jahren nach der Synode zeigte sich bald, dass die Kirche vom Unerwarteten zum Erwartbaren zurückzukehren bemüht war, und so fielen auch viele Medien in den konventionellen Stil der Aktualitäts-

6 Michael Schmolke: Kirche und gesellschaftliche Kommunikation. In: Dieter Emeis/ Burkard Sauermost (Hg.): Synode - Ende oder Anfang. Düsseldorf 1976, S. 303-315, hier S. 305.

7 Eckhard Bieger: Das Öffentlichkeitsdilemma der katholischen Kirche. Mainz 2003, S. 155 . 
findung zurück und holten sich ihren Stoff wieder aus bewährten Zonen: Personalismus, Negativmeldungen und Konflikt.

Bestimmte Entwicklungen kamen ihnen dabei entğegen. Ich nenne nur drei, auf die ich später noch zurïckkommen werde:

- die Wahl des polnischen Papstes, der sich programmatisch Johannes Paul nannte, den Beweger Johannes XXIII. wie den Formensicherer Paul VI. in seinen Namen und sein Programm aufnehmend;

- die Personalpolitik des Vatikans, insbesondere die Bischofsernennungen, und

- die Missbrauchsaufdeckungen in den USA und einigen anderen Ländern.

Auf diese Weise blieb die Kirche ständig Medienthema, ohne dass es eigener Medien dazu bedurft hätte. „Publik“ war tot, bevor man hätte erproben können, was eine deklariert katholische Wochenzeitung in dieser Gesellschaft hätte bewirken können; der "Rheinische Merkur" wollte trotz erheblicher Geldspritzen und der Vereinigung mit „Christ und Welt" nicht wirklich gedeihen; die „Deutsche Tagespost" versorgte ihren gefestigten Leser-Sektor am konservativen Rand, und die Diözesanblätter dümpelten nicht einmal dahin: in den 30 Jahren von 1963 bis 1993 hatte sich ihre Gesamtauflage von rund 2,4 auf 1,2 Millionen halbiert. Im Jahr 2004 lag die verkaufte Auflage nur noch bei 875000 Exemplaren. ${ }^{8}$ Ganz abgesehen davon war ja schon in den sechziger Jahren klar, dass sie für eine Vertretung kirchlicher Anliegen gegenüber der Gesamtöffentlichkeit nicht taugten, sonst hätte man ja „Publik“ nicht gegründet.

\section{Die Kirche als eine unter anderen „gesellschaftlich relevanten Gruppen"}

Was bei all den Klagen und selbstkritischen Analysen allenfalls am Rande erwähnt wurde, in der Regel aber unbeachtet blieb, war die Tatsache, dass auch die pressebewusste SPD in der gleichen Zeit ihre letzten parteigebundenen Tageszeitungen und am Ende sogar ihre Führungszeitschrift verloren hatte. Und dem mächtigen DGB war es genau so ergangen. Weder die Gewerkschaft noch die SPD verschwanden deswegen aus der Öffentlichkeit - und die Kirche auch nicht.

Jürgen Habermas hatte 1962 auf den Punkt gebracht, was frühe PRForscher schon länger wussten: Öffentlichkeit stellt sich her, etwa durch die Relevanz von Ereignissen oder Personen, aber Öffentlichkeit ist 
durchaus auch herstellbar, indem man Öffentlichkeitsarbeit betreibt. ${ }^{9}$ In den ersten beiden Nachkriegsjahrzehnten war die Kirche wie selbstverständlich davon ausgegangen, dass sie das Lebensprinzip der Gesellschaft sei, und zwar auf privilegierten Grundlagen. Im Westen Deutschlands war die wilhelminisch-protestantische Zweiheit von Thron und Altar durch Frings und Adenauer ersetzt worden. Die Kirche sei keine Partei, keine Gewerkschaft, kein Verein, brachte mir der eingangs erwähnte Chefredakteur bei, und deshalb dürfe man die Gläubigen auch nicht als „Mitglieder" der Kirche bezeichnen, genau so wenig, wie man von Kirchen im Plural schreiben und reden dürfe.

Die Gesellschaft aber, die man jetzt gern eine pluralistische nannte, sah das anders. Aus ihrer Sicht waren die katholische wie die sonstigen Kirchen und Religionsgemeinschaften nichts anderes als „gesellschaftlich relevante Gruppen". Die Zusammensetzung der meisten deutschen Rundfunkräte spiegelte diese beinahe endgültige Säkularisierung: In ihnen saßen und sitzen die Vertreter der Kirchen neben jenen der Gewerkschaften und Kammern, der Bauern- und Bühnenverbände, der Wissenschaft und der Erwachsenenbildung usw. usw.

Reste einer privilegierten Stellung finden wir allenfalls dort, wo den Kirchen die Verantwortung für sogenannte Verkündigungssendungen eingeräumt ist. Nicht zuletzt deshalb haben beide Konfessionen gezögert, die Liberalisierung bzw. Dualisierung des Rundfunks aus voller Überzeugung zu begrüßen. Denn bei den Öffentlich-Rechtlichen wusste man, was man hat, bei den neu entstehenden privatrechtlichen Radio- und Fernsehanbietern konnte man allenfalls auf ungewisse Chancen setzen.

\section{Pastorale Argumente für die Kirchenpresse}

Während die Parteien und Gewerkschaften sich den Verzicht auf im Vollsinne, d.h. ideologisch und ökonomisch, eigene Massenmedien allenfalls von Traditionsargumenten schwer machen ließen, stand und steht die Kirche vor einem echten Dilemma. Sie betrachtet ihre Medien, also in der Hauptsache die Kirchenpresse, stets auch als ein Instrument der Seelsorge. Diese Vorstellung hatte in der Entstehungszeit jener Pressegattung, also im 19. Jahrhundert, einen anderen Akzent als heute. Damals ging es um die apologetische Funktion: Gegen eine Welt von Feinden, hießen diese nun Liberalismus oder kämpferischer Protestantismus oder „die Juden“ oder Bismarck, musste man öffentlich die Stimme erhe-

9 Jürgen Habermas: Strukturwandel der Öffentlichkeit. Neuwied, Berlin 1962. 
ben, die Wahrheit verteidigen können, um dadurch die eigene Herde beieinander zu halten.

20 Jahre nach dem Zweiten Weltkrieg stand ein anderes Argument im Vordergrund: Angesichts sinkender Kirchenbesucherzahlen hoffte man jene, die von der direkten mündlichen Verkündigung, also von der Kanzel, nicht mehr erreichbar waren, weiterhin per Wochenblatt ansprechen zu können. Dass diese Hoffnung trog und trügt, geht aus wiederholten Analyseergebnissen hervor: Kirchenblattleserschaft und die Teilmenge der treuen Gottesdienstbesucher dürften fast deckungsgleich sein; deshalb gibt es seit einigen Jahren Überlegungen, ob man nicht besser alle Kirchenmitglieder mit Gratis-Verteilblättern zu erreichen versuchen sollte. Meines Wissens hat das nur die Erzdiözese Wien seit 1997 in großem Stil versucht (Magazin „Dialog“) ${ }^{10}$, die Aktion aber mit Ende 2003 wieder eingestellt.

Für die Anhänglichkeit, man kann auch sagen: Treue, speziell der deutschen Diözesen zu ihren Bistumsblättern gab es aber, jedenfalls in der Zeit des Wiederaufbaus, noch einen Anknüpfungspunkt, der aus der Unterdrückung der konfessionellen Presse durch die nationalsozialistische Presselenkung herrührte: Der Begriff Bistumsblatt, d.h. das Prinzip eine Diözese - ein Bischof - eine Zeitung ist ja 1935/36 von den Unterteufeln des Propagandaministers erfunden worden, um die Vielfalt der frommen Blättchen abwürgen zu können. ${ }^{11}$ Eben dieser Druck hat $z \mathfrak{u}$ einer starken Solidarisierung der Gläubigen mit den Resten ihres Blätterwalds geführt, und nachdem diese 1941 auch noch endgültig unterdrückt worden waren, war es für sie nicht schwer, unter der alliierten Lizenzpolitik mit weißer Weste als Bistumsblätter wieder zu erstehen. Damit hatten die Bischöfe ein für die damaligen Verhältnisse hoch modernes Kommunikationsinstrument in der Hand: eine Monopolzeitung, die trotzdem gern akzeptiert wurde; sie zu abonnieren, war auch ein „Zeichen des Glaubens" und einer neuen Gesinnung. Innerhalb der Diözese war das Blatt konkurrenzlos, und die Erträge konnten sich sehen lassen.

Weil dieser Pressetyp funktional modern war, verzichtete man $20 \mathrm{Jah}$ re lang auf Modernisierungen, und erst in den sechziger Jahren, als die Auflagen sanken und die Funktionalität fraglich geworden war, begann man mit äußeren Modernisierungen in größerem Umfang. Aber die Abonnenten gingen nicht von der Fahne, weil der Umbruch vorher altmodisch gewesen war, sondern weil sie nach und nach dahin starben. Ihre poten

10 Erich Leitenberger: Das Experiment „Dialog“. In: ComSoc, 30. Jg. 1997, S. 273-277.

11 Vgl. Michael Schmolke: Die kirchlich-konfessionelle Presse. In: Jürgen Wilke (Hg.):

Mediengeschichte der Bundesrepublik Deutschland. Köln 1999, S. 350-374, hier S. $350 f$. 
tiellen Abo-Erben aber wuchsen in einer Welt heran, die dem Strukturwandel der Öffentlichkeit so intensiv unterworfen war wie nie eine Generation vor ihnen.

\section{Das Fernsehen - oder: Aufmerksamkeit wird knapp}

Seit Mitte der 1950er Jahre wurde der Fernseher ein „nöthiges Möbel im Hause" - so hatte Adolph Kolping 100 Jahre zuvor die katholischen Periodika genannt. Entgegen anfänglichen Befürchtungen verdrängte er die Printmedien nicht - im Rückblick möchte man sagen: im Gegenteil, er begleitete oder bewirkte sogar ihren Wirtschaftswunder-Aufstieg. Wohl aber veränderte das Fernsehen den Rezeptionsstil, ganz allgemein, besonders jedoch in den Familien. Die auf Niklas Luhmann zurückgeführte Redewendung "Aufmerksamkeit ist knapp" bekam, bevor sie geprägt wurde, eine sehr reale Bedeutung: Der Bundesbürger ab 14 Jahre verbrachte fortan (im statistisch errechneten Durchschnitt) pro Tag plus-minus zwei Stunden vor dem Fernsehgerät. Das war der quantitative Aspekt. Der qualitative Aspekt bedeutete, dass er dort Medieninhalte, und zwar in Gestalt bewegter Bilder, ins Haus geliefert bekam, die ihm weder die großen Illustrierten noch der "Spiegel“ noch die Tagespresse anbieten konnten - und schon gar nicht die Kirchenpresse. Tagespresse und "Spiegel“ hatten Funktionen, auf die sehr viele Menschen nicht verzichten konnten. Deshalb wurden sie vom Fernsehprogramm zwar herausgefordert, aber nicht bedroht. Anders sah es schon bei den großen Illustrierten aus: $Z$ wei von den Großen Vier verschwanden vom Markt.

Die Kirchenzeitungen erbrachten keine unabdingbaren Leistungen; sie wurden auf dem Markt gehalten, weil ihre Herausgeber es so wollten; ihre Chance, einen erheblichen Teil vom Aufmerksamkeitskuchen für sich abzuschneiden, sank jedoch erheblich. Experten waren sich einig, dass sie am Bedarf vorbeiproduzierten; böse Zungen sagten, dass sie Fragen beantworteten, die niemand stellte.

\section{Essentials des kirchlichen Verhältnisses zur Öffentlichkeit}

Als man dann das Experiment „Publik" startete, war das auch die Probe aufs Markt-Exempel. Dass sie nicht bestanden wurde, lag nachgewiesenermaßen an Managementfehlern. ${ }^{12}$ Aber es lag ebenso sehr an

12 Otto B. Roegele: „Publik“ - ein Lehrstück. In: Petra E. Dorsch-Jungsberger/Walter Hömberg/Walter J. Schütz (Hg.): Otto B. Roegele: Plädoyer für publizistische Verantwortung. Konstanz 2000, S. 269-295. 
zwei Verfassungs- oder auch Konstruktionseigentümlichkeiten, die der römischen Kirche von Anfang an eingestiftet sind.

1. Die Kirche kann hinter ihren Verkündigungs-, und das heißt auch: hinter ihren pastoralen Auftrag nicht zurück. Ließe sie davon ab, wäre sie nicht mehr diese Kirche.

2. Die Kirche ist nicht demokratisch verfasst. Ihre hierarchische Verfassung ist jedoch, wie die Geschichte lehrt, durchaus funktionstüchtig. Mit dem Stichwort Dialog ist im Zweiten Vaticanum eine elegante Einflugschneise für demokratische Modernisierungselemente eröffnet worden, die der traditionsgeheiligten Verfassung eine neue Dynamik verliehen haben. Räte und Beiräte gibt es in Fülle, auf unterer Ebene sogar Wahlen. Aber die Souveränität geht nicht vom Volke aus, kann gar nicht vom Volke ausgehen.

Unternehmensberater würden diese beiden Eigentümlichkeiten, nämlich unveränderbare Auftragsbindung und hierarchische Verfassung, zunächst einmal Konstruktionsfehler nennen. Der vorherige Papst hat der staunenden Welt vorgeführt, welche Kraft ihnen innewohnt.

a) Er hat mit allen geredet, aber alle Entscheidungen aus der Autorität seines singulären Amtes getroffen.

b) Er hat bestimmte Inhalte der Doktrin immer wieder öffentlich bekräftigt und sich kein Jota wegstreichen lassen, wenn sie angefochten wurden.

Beides ist vielen Gläubigen und besonders vielen Theologen auf den Nerv gegangen, hat aber so manche Journalisten fasziniert. Von ihnen kommt das ambivalente Urteil: Johannes Paul sei zwar, wo es um die Realitäten der Gesellschaft gehe, hinter dem Mond gewesen; aber als Medienperson sei er ein ganz außergewöhnlicher Glücksfall.

In der Tat gehören - quasi von Natur aus - wesentliche aktualitätsstiftende Eigenschaften zu seiner Person:

- das Unerwartete: von Anfang an - er war nicht Italiener, sondern Pole;

- der Konflikt: die Stichworte Zölibat, viri probati und Priesterweihe für Frauen mögen genügen;

- das Menschliche: auch hier nur Beispiele: das Attentat, die schwere Krankheit und die Art, wie er mit ihr umgeht.

\section{Der Aktualitätspapst und seine Personalpolitik}

Die vatikanische Personalpolitik der letzten zwei Jahrzehnte sorgte für ein weiteres Aufmerksamkeitsfeld. Nicht dass die Ernennungen konservativer Bischöfe unerwartet gewesen wären. Ein Papst, der wieder und wieder deutlich machte, dass er hinter eine bestimmte Grenze nicht 
zurückzugehen gedenke, wird für aufgeweichtes Terrain - dazu gehören Deutschland, Österreich und die Niederlande - konservative Bischöfe bestellen. Das Unerwartete kam von dem einen oder anderen der so ins Amt gekommenen Hirten. Sie erwiesen sich nicht nur, was zu erwarten war, als Hardliner, sondern auch als Bekenner dieser ihrer Linie. Dabei zeigte sich, dass z.B. Johannes Dyba von Fulda und Kurt Krenn von St. Pölten so wie ihr oberster Chef Medientalente waren, und zwar nicht nur, wenn und weil sie ins Fettnäpfchen traten, sondern weil sie sich auch in Interviews und Talkshows nicht bremsen ließen und auf der Aktualitätsschiene Konflikt erheblich zum Infotainmentwert solcher Sendeformate beitrugen.

Folgerichtig wurden sie häufiger um Interviews gebeten und in einschlägige Sendungen eingeladen.

Während Dyba zwar oft getadelt wurde, aber am Ende ohne Tadel schied, führte sich Krenn durch die Skandale um sein Priesterseminar selbst der medialen Zweitverwertung zu: Er wurde zum „Fall“ und kam $z \mathfrak{u}$ Fall. Wasser zu predigen und Wein getrunken $z \mathfrak{u}$ haben, war ja schon seinem Gesinnungsbruder Hans Hermann Groer, dem Kardinal-Erzbischof von Wien vorgeworfen worden, bis er ruhmlos aus dem Amt schied wie jetzt Kurt Krenn.

Beide haben dem Ansehen der Kirche schweren Schaden zugefügt, sodass man nur mehr auf das Motto des "Osservatore Romano“ setzen kann: non praevalebunt. An beiden hat sich aber auch gezeigt, wie stark das Interesse der Massenmedien an der Institution ist, die da eine feste Burg, aber auch das Haus voll Glorie genannt wird. Nach meiner Einschätzung ist das nicht nur das tagesgebundene Interesse am Skandalon, sondern auch die existenzielle Sorge angesichts des Schwächelns der Institution, die weiterhin als Pfeiler einer geordneten Welt gesehen wird.

Die aktuelle Diskussion um Fundamentalismus und - im Speziellen Islamismus stützt meine These. Mit erhöhter Aufmerksamkeit schaut man auf transzendierende Lehren, seitdem der Kommunismus als innerweltliches Heilsversprechen, aber auch als Herausforderung des Liberalismus abgewirtschaftet hat. Seither muss, wenn es um außermaterialistische Zielsetzungen geht, der pragmatische Kapitalismus mit ideologischen Leihgaben aufgerüstet werden. George Bush holt sie sich aus einem patriotistisch angehübschten Weltmachtdenken ebenso wie aus einem holzgeschnitzten Christentum.

Der Rest der Welt fühlt sich nicht gut dabei, dies umso stärker, weil der islamische Teil dieses Restes der Welt sich ganz besonders schlecht fühlt und aus sèinen heiligen Schriften Handlungsanleitungen gewinnt, wie sie vor 1000 Jahren unter christlichen Vorzeichen die Kreuzzüge inspiriert haben. 
Die Bergpredigt richtet zur Zeit in der Praxis wenig aus, weder gegen den entfesselten Global-Kapitalismus noch gegen den ebenso globalen Terrorismus. Umso aufmerksamer wird die Theorie beobachtet, die von geistlichen Zentren ausgeht; stets wird sie kritisch beobachtet, oft, wenn sie fehlschlägt, mit einer gewissen Schadenfreude. Aber missen möchten unsere meinungsbildenden Leitmedien sie nicht. Die Kirchen- und Religionsberichterstattung füllt in FAZ, „Welt" und „Süddeutscher Zeitung“ viele Spalten. Sie werden in der Regel von gut ausgebildeten Mitarbeitern geschrieben, die auch skandalisierten Hauptpersonen einen fairen Prozess angedeihen lassen.

\section{Image auf Kredit}

Blicke ich auf diese, die höhere Hemisphäre der deutschsprachigen Medienwelt - die regionale Abonnementspresse und die öffentlich-rechtlichen Rundfunkanstalten gehören eigentlich auch dazu -, so habe ich eine Antwort auf die Frage, die mir der Präsident des Bayerischen Presseclubs gestellt hat: Warum die Kirche, das wollte er gefragt wissen, „es nicht schafft, ihr gesellschaftliches und mediales Image nachhaltig $\mathrm{zu}$ verbessern". Fast jedes Image kann verbessert werden, aber das Image der Kirche ist gar nicht so schlecht. Der Kredit, den Konzil und Synode geschaffen haben, ist noch keineswegs verbraucht. Die Offenheit, mit der sich, wenn nicht alle, so doch manche Bischöfe den demütigenden Skandalen gestellt haben, ist nicht ohne Eindruck geblieben. Die Tatsache, dass die Kirche nicht mehr vorgibt, in allem und jedem una voce zu sprechen, wird honoriert ebenso wie die Tapferkeit der Regionen gegenüber der global denkenden Zentrale. Superkonservative Zirkel werden eher um ihres Kuriositätswertes willen beobachtet und - nach meinem Eindruck - nicht überbewertet. Die durch und durch hämische Berichterstattung ist in das eine oder andere Nachrichtenmagazin abgewandert.

Das Gesamtbild, wie es sich mir darstellt, ist ohne nennenswerte aktive Mitwirkung der offiziösen Kirchenpresse entstanden. Aber keine Kirchenblattredaktion würde heute noch Vorkommnisse wie jene in St. Pölten zu bemänteln versuchen. Die Zeiten, da manche insbesondere diözesan oder theologisch brisante Themen überhaupt nicht angesprochen werden durften, sind (fast) vorbei.

Insofern hat die seit der Synode laufende Reformdiskussion eine ganze Menge bewirkt, und zwar auch durch jene Veränderungen, die damals angestoßen wurden:

- Die Deutsche Bischofskonferenz ist sich ihrer Sprecherverantwortung bewusst, und ihr Vorsitzender macht seine Sache gut. 
- Die Diözesen haben eingesehen, dass Öffentlichkeitsarbeit in dieser Gesellschaft eine Notwendigkeit ist.

- Die KNA funktioniert wie eine Nachrichten- und nicht wie eine PR-Agentur.

- Die gezielte Nachwuchsförderung, ohne Scheuklappen in den Ausbildungsangeboten, hat Früchte getragen.

- Die Journalistik-Ausbildung an der Katholischen Universität Eichstätt findet hohe fachliche Anerkennung.

- Die Kirchenpresse ist durch mancherlei Reformen, die auch in der Gegenwart nicht nachlassen ${ }^{13}$, ansehnlich geworden. Dass immer weniger Menschen sie ansehen wollen, steht auf einem anderen Blatt.

$\mathrm{Zu}$ diesem anderen Blatt, auf dem keineswegs nur von der kirchlichen Presse die Rede ist, möchte ich im vorletzten und im letzten Abschnitt etwas sagen.

\section{Bilder als Vorbilder für die Lebensgestaltung}

Kirchen und andere Religionsgemeinschaften sehen eine ihrer Hauptaufgaben darin, ihren Anhängern, eigentlich aber allen Menschen, gewisse Regeln für die Lebensgestaltung vorzugeben. In einer aus Konsens hervorgegangenen Kernform tun dies auch Staatsverfassungen und staatliche Gesetze. Deren Geltung kann bis zu einem gewissen Ausmaß mit Hilfe der Staatsgewalt durchgesetzt werden. Für die Regelungsbemühungen der Kirche gilt das schon lange nicht mehr. Der Papst hat keine Divisionen, und er würde es heute aus pastoraler Überzeugung weit von sich weisen, welche haben zu wollen.

Vorbilder für die Lebensgestaltung werden heute zum guten Teil aus den Massenmedien bezogen, ungeachtet der Tatsache, dass deren Verantwortliche beteuern, dass sie nicht angetreten sind, um Vorbilder zu liefern. Sie berichten das, was geschieht oder was gesagt wird, und sie unterhalten Menschen mit erdichteten Geschichten: fiction. Die Geschichten stammen zum Teil aus der Phantasie, zum Teil lehnen sie sich an das „wirkliche Leben“ an. In vielen von ihnen siegt ganz am Ende das Gute oder es wird doch etwas erkennbar, was man einen fernen Abglanz der Gebote oder der Gesetze oder der Wahrheit nennen könnte. Dieser Abglanz kann, wie wir gelernt haben, auch durch Fälschungen hergestellt

13 Vgl. dazu das von Helmut Haimerl in diesem Heft vorgestellte Reformkonzept der Mediendienstleistung GmbH, erstellt im Auftrag der Deutschen Bischofskonferenz. 
werden. Clausewitz hat zu Beginn des 19. Jahrhunderts geschrieben: „Die meisten Nachrichten sind falsch ..." Wir heutigen wissen: Die meisten Nachrichten können gefälscht sein.

Ähnliches gilt für den Sektor fiction. Hier wissen wir, dass das, was wir sehen, fingiert ist - dennoch kann es uns eine Wahrheit nahe bringen. Am weitesten weg von Nachrichten-Echtheit und Fictions-Wahrheit sind jene „Formate“, in denen echte und wirkliche Menschen dazu gebracht werden, sich selbst, ihren Mitspielern und ihrem Publikum ihr Leben so vorzuspielen, wie es in Wirklichkeit nicht ist. Am Ende erwächst aus diesem Prozess eine entfesselte Lebensbeichtenbekennerin oder ein „Superstar". Der Superstar ist eine Fälschung. Die meisten Geschichten sind falsch. Selbst der australische Urwald, aus dem Fernsehberühmtheiten angeblich herausgeholt werden möchten, ist - in diesem Falle - falsch. Aber die aus dem Prozess der Serie erwachsenden Anhängerschaften sind echt. Ihr durch Vorbilder geprägter Lebensstil, ihr Lebensgefühl, ihre Werte werden tagtäglich im „wirklichen Leben“ zur Schau getragen - nicht von allen, aber von vielen. Sie spielen vorfabrizierte Rollen und wähnen sich auf dem Weg nach oben.

\section{Glaubwürdigkeit als Chance}

Der Präsident des Bayerischen Presseclubs fragt nach den „Möglichkeiten, die der kirchlichen Publizistik unter den derzeitigen Bedingungen noch bleiben, um eine journalistisch verantwortete und das Zielpublikum erreichende Arbeit zu leisten". Es bleiben, wenn ich es ehrlich sagen darf, nicht viele Möglichkeiten, es sei denn, die kirchliche Publizistik bescheidet sich mit einem sehr eng definierten Zielpublikum. Selbst wenn es gelänge, die kirchlichen Printmedien von heute auf morgen in Fernsehstationen umzuwandeln, die Chancen blieben gering, denn „das Fernsehen“, wie es heute ist, zeigt sich - ich zitiere Dieter Stolte - „als Verstärker und Beschleuniger eines Zeitgeistes, dem ,Brot und Spiele“ wichtiger sind als die Wahrnehmung ethischer Verhaltensweisen in der Gesellschaft".

Auf der Brot und Spiele-Ebene können christlich fundierte Medien nicht mitmischen. Auf der Ebene der formalen Modernisierung haben sie es mit großem Aufwand versucht. Auf der inhaltlichen Ebene dürfen sie um keinen Preis mit den Wölfen heulen. Dadurch verlören sie das, was sie vielen anderen Medien voraushaben: ihre Glaubwürdigkeit.

Aber auch der Rückzug in innerkirchliche oder konservative oder elitäre Gettos eröffnet keine neuen Möglichkeiten. Publizistik will in der Öffentlichkeit gehört werden und nicht nur in der Kerngemeinde. 
Glaubwürdigkeit ist eine unique selling proposition. Wir schreiben sie, manchmal leichtfertig, den prestige papers zu. In puncto Glaubwürdigkeit sollte die christlich fundierte Publizistik alles daran setzen, zur PrestigeKlasse zu gehören.

Alles daran setzen heißt: dem Nachwuchs eine solide Ausbildung ermöglichen, den erfahrenen Profis anspruchsvolle Weiterbildung; heißt auch: inhaltlich und formal nicht und niemals mit den Wölfen heulen, nicht mitwirken beim Verschweigen noch beim Beschönigen.

Wie kostbar Glaubwürdigkeit ist, haben wir in den letzten Jahren erlebt, als die Glaubwürdigkeit der Institution Kirche durch Skandale anfechtbar wurde.

Die Glaubwürdigkeit eines Mediums oder einer Mediengattung erwächst nicht automatisch aus dem Gründungsprogramm oder den Richtlinien der Herausgeber. Glaubwürdigkeit erwächst aus dem Sachwissen, den professionellen Fähigkeiten und der Redlichkeit der Menschen, die für ein Medium arbeiten. Glaubwürdigkeit ist eine Tugend und eröffnet Möglichkeiten, die von Marktanalysen nicht erfasst werden. 